${ }^{\circ}$ Entomologica Fennica. 3.XII.1990

\title{
Typenrevision der von Hellén beschriebenen Cryptinae (Hymenoptera, Ichneumonidae)
}

\author{
Klaus Horstmann
}

Horstmann, K. 1990: Type revision of the species of Cryptinae described by Hellén (Hymenoptera, Ichneumonidae). — Entomol. Fennica 1:181-187.

The types of 38 taxa of the subfamily Cryptinae (= Gelinae $=$ Phygadeuontinae) described by Wolter Hellén are revised. Two new synonyms are indicated: Phygadeuon austriacus (Gravenhorst), syn. Iselix elfvingi Hellén, and Hedylus crassicornis Ashmead, syn. Aclastus setosus Hellén. Lectotypes are designated for four species. A new name, Hemiteles rufigaster, is substituted for the preoccupied name Hemiteles rufiventris Hellén. The male described by Hellén under the name Idiolispa morio is regarded as a separate species, Hidryta atlantica sp. n.

Klaus Horstmann, Zoologisches Institut der Universität Würzburg, Röntgenring 10, D-8700 Würzburg, FRG

Wolter Hellén hat 38 Arten und Formen der Unterfamilie Cryptinae neu beschrieben, die Mehrzahl davon in seiner umfangreichen Arbeit über die Kollektivgattungen Phygadeuon Gravenhorst und Hemiteles Gravenhorst (Hellén 1967), die anderen verstreut in verschiedenen Publikationen. Nachdem ich begonnen hatte, mich für die europäischen Arten von Hemiteles s. 1. zu interessieren, habe ich schon bald mit Hellén selbst Kontakt aufgenommen und den größeren Teil der Typen seiner Arten noch zu seinen Lebzeiten untersuchen können. Notizen darüber sind in verschiedenen eigenen Publikationen enthalten, außerdem hat Townes (1983) aufgrund meiner Hinweise einige Arten in seine Revisionen eingearbeitet. In den letzten Jahren konnte ich diese Typenrevisionen vervollständigen und lege hier eine Übersicht über alle von Hellén beschriebenen Arten und Formen vor. Nur die Typen dreier Taxa, die von Sawoniewicz (1978) und Jussila (1979) revidiert worden sind, habe ich nicht noch einmal untersucht.

Die Typen aller untersuchten Taxa (mit einer Ausnahme: Aclastus caudator) befinden sich im Zoologischen Museum der Universität in Helsinki.

\section{Revisionen}

Asyncrita canaliculata Hellén

Asyncrita canaliculata Hellén, 1944:12. Lectotypus (O) von Jussila (1979:29) festgelegt (nicht untersucht).

Gültiger Name: Atractodes foveolatus (Gravenhorst, 1829) (Jussila 1979:29). 


\section{Asyncrita scutellata Hellén}

Asyncrita scutellata Hellén, 1944:13. Holotypus (Q) von Jussila (1979:30) beschriftet (nicht untersucht).

Gültiger Name: Atractodes scutellatus (Hellén, 1944) (Jussila 1979:30).

\section{Idiolispa morio Hellén}

Idiolispa morio Hellén, 1949:5f. Lectotypus (૧) von Zwart beschriftet und hiermit festgelegt: "Gr. Canaria, Atalaya, R. Storå", "210".

Gültiger Name: "Trychosis" nigriventris (Habermehl, 1918) (Aubert 1969:51; Schwarz 1988:54).

Die Literaturangaben über diese Art widersprechen einander, was darauf zurückzuführen ist, daß Hellén seine Art nach gemischtem Material beschrieben hat. Der Lectotypus ( $)$ ) von den Kanaren gehört zu der Art, die bereits von Habermehl (1918:152) und Seyrig (1935:181) unter den Namen Idiolispa analis (Gravenhorst) forma nigriventris Habermehl beziehungsweise Idiolispa vagabunda Seyrig von den Kanaren beschrieben worden ist. Aubert (1969:51) hat diese Taxa aufgrund der übereinstimmenden Beschreibungen und Fundorte als erster synonymisiert. Ein Paralectotypus $\left(\sigma^{\top}\right)$ von den Azoren gehört zu der Gattung Hidryta Förster. Dieser Typus wurde von Ortega \& Baez (1980:84 ff.) untersucht, die daraufhin I. morio $\left({ }^{\top}\right)$ ganz richtig als eigene Art neben I.vagabunda angesehen, die unterschiedliche Gattungszugehörigkeit beider Arten aber nicht bemerkt haben. Diese Hidryta-Art wird in einem Anhang neu beschrieben.

Die Determination der Art von den Kanaren (nigriventris Habermehl) nach den publizierten Bestimmungsschlüsseln führt zu der Gattung Trychosis Förster. Zu dieser Gattung bestehen aber einige Unterschiede. Herr Drs. K. W. R. Zwart beabsichtigt, über dieses Problem in Kürze zu publizieren.

\section{Habrocryptus canariensis Hellén}

Habrocryptus canariensis Hellén, 1949:6. Lectotypus (Q) hiermit festgelegt: "Tenerife, Tacoronte, R. Frey", "4766".

Gültiger Name: Ischnus canariensis (Hellén, 1949) (Ortega \& Baez 1980:90f.)

\section{Brachycephalus tenerifae Hellén}

Brachycephalus tenerifae Hellén, 1949:7. Lectotypus (o) von mir beschriftet und von Ortega \& Baez (1980:70) festgelegt: "Tenerife, Tacoronte, R. Frey", "2916".

Gültiger Name: Dichrogaster tenerifae (Hellén, 1949) (Ortega \& Baez 1980:70; Townes 1983:115) .

Brachycephalus tenerifae Hellén var. thoracicus Hellén

Brachycephalus tenerifae Hellén var. thoracicus Hellén, 1949:7. Holotypus (ф): "Tenerife, Orotava, R. Frey", "3171".

Gültiger Name: Dichrogaster tenerifae (Hellén, 1949) (Townes 1983:115).

\section{Hemiteles rufiventris Hellén}

Hemiteles rufiventris Hellén, 1949:7f. Praeoccupiert durch Hemiteles rufiventris Brullé, 1846. Lectotypus (o) von mir beschriftet und von Ortega \& Baez (1980:81) festgelegt: "Tenerife, Agua Garcia, R. Frey", "306".

Gültiger Name: Hemiteles rufigaster nom. nov.

Die Art ist Hemiteles similis (Gmelin) ähnlich, unterscheidet sich aber von dieser durch folgende Merkmale: Postpetiolus dorsal auf der Apicalhälfte nicht gestreift; zweites und drittes Gastertergit dorsal median unpunktiert und unbehaart, an den Rändern behaart, mit relativ langen, zerstreut stehenden Haaren; Beine und Gaster von der Spitze des Postpetiolus an hell rotbraun.

\section{Hemiteles speciosus Hellén}

Hemiteles speciosus Hellén, 1949:8. Lectotypus (@) von Zwart beschriftet und hiermit festgelegt: "Tenerife, Orotava, R. Frey", "1556".

Gültiger Name: Gelis speciosa (Hellén, 1949) (Horstmann 1986:398, 404).

G. speciosa ist unter den verwandten Arten der Gelis areator -Gruppe charakterisiert durch: Area superomedia so lang wie oder etwas länger als breit, innen glänzend, sehr zart gekörnelt oder glatt.

\section{Atractodes nigripennis Hellén}

Atractodes nigripennis Hellén, 1949:8. Lectotypus (o) hiermit festgelegt: "Tenerife, Los Mercedes, R . Frey", "3038".

Gültiger Name: Atractodes nigripennis Hellén, 1949. 
Ischnus porrectorius (Fabricius) var. melanarius Hellén

Ischnus porrectorius (Fabricius) var. melanarius Hellén, 1957:131. Holotypus (o): "H:fors Hellén”, "3786".

Gültiger Name: Caenocryptus melanarius (Hellén, 1957)'.

Die Art ist mit den publizierten Bestimmungsschlüsseln nicht determinierbar.

Apsilops cinctorius (Gravenhorst) var. tenebrosus Hellén

Apsilops cinctorius (Gravenhorst) var. tenebrosus Hellén, 1957:133. Holotypus (o): "Parikkala Hellén”, "1303”. Gültiger Name: Apsilops tenebrosus Hellén, 1957.

Der Holotypus weicht von den mir aus Mitteleuropa bekannten Weibchen von A. cinctorius (Gravenhorst) durch folgende Merkmale ab: Körperlänge $5 \mathrm{~mm}$; Postnervulus bei 0.7 seiner Länge gebrochen, der vordere Ast sehr schräg nach innen gestellt; Bohrerklappen anscheinend etwas länger, 0.8 mal so lang wie die Hintertibien; Scutellum schwarz; Femora und Tibien der Hinterbeine jeweils apical dunkel gezeichnet.

\section{Ischnurgops pellucidator (Gravenhorst) macula-} tus Hellén

Ischnurgops pellucidator (Gravenhorst) maculatus Hellén, 1957:139. Holotypus (o) von Sawoniewicz (1978:129) beschriftet (nicht untersucht).

Gültiger Name: Bathythrix maculata (Hellén, 1957) (Sawoniewicz 1978:129).

Bei der Beurteilung des Ranges (subspezifisch oder infrasubspezifisch) der von Hellén benannten Form gibt es Interpretationsschwierigkeiten, die auf Ungenauigkeiten der Autoren zurückzuführen sind. Entgegen seiner sonstigen Gewohnheit gibt Hellén (1957:139) nicht an, ob er seine Form als Varietät oder als Aberration verstanden wissen

\footnotetext{
' In seiner ersten größeren Publikation hat Hellén (1915: 7) definiert, daß er unter "Varietät" eine Form versteht, "die durch bedeutetende Verschiedenheiten von der Hauptart abweicht und somit meist als biologische oder geographische Rasse zu betrachten ist." Demgegenüber sieht er "Aberrationen" als individuelle Abweichungen an. Deshalb werte ich Helléns Varietäten als Taxa von subspezifischem Rang.
}

will, sondern führt den Namen ohne nähere Bezeichnung als dritten Namen eines Taxons ein. Sawoniewicz (1978:129) zitiert das Taxon irrtümlich so, als ob Hellén es als Aberration bezeichnet habe, verwendet den Namen aber doch als verfügbaren Namen für eine Art. In einer zweiten Publikation (Sawoniewicz 1980:328) berichtigt er stillschweigend diese Inkonsequenz. Aubert (1981:18) schließlich greift Sawoniewicz an (vermutlich nur dessen Publikation von 1978), stützt sich aber in seiner Interpretation nicht auf die Nomenklaturregeln. Meines Erachtens ist der Name Helléns nach den Nomenklaturregeln (Artikel 45f(i) in der Fassung von 1985) verfügbar, ohne daß dabei ein Ermessensspielraum besteht.

\section{Phygadeuon lateareolatus Hellén \\ Phygadeuon lateareolatus Hellén, 1967:88f. Holotypus (o): "Kuvernööri", "M. Hellén”, "1141". \\ Gültiger Name: Phygadeuon lateareolatus Hellén, 1967.}

\section{Phygadeuon striiventris Hellén}

Phygadeuon striiventris Hellén, 1967:89f. Holotypus (o): "Kuolajärvi Kurtti Hellén”, “4838”.

Gültiger Name: Phygadeuon striiventris Hellén, 1967.

\section{Iselix elfvingi Hellén}

Iselix elfvingi Hellén, 1967:91. Holotypus (○): "Kuopio, R. Elfving, 8.6.1960".

Gültiger Name: Phygadeuon (Iselix) austriacus (Gravenhorst, 1829) (syn. nov.).

\section{Phyzelus boreaphilus Hellén}

Phyzelus boreaphilus Hellén, 1967:93f. Holotypus (o): "Ivalo", "Hellén", "891".

Gültiger Name: Theroscopus boreaphilus (Hellén, 1967).

\section{Phyzelus ponojensis Hellén}

Phyzelus ponojensis Hellén, 1967:94. Holotypus (q): "Ponoj", "Hellén", "158".

Gültiger Name: Phygadeuon ponojensis (Hellén, 1967) (Horstmann 1975:104).

\section{Phyzelus inaris Hellén}

Phyzelus inaris Hellén, 1967:95. Holotypus (@): "Enari”, "B. Poppius".

Gültiger Name: Phygadeuon inaris (Hellén, 1967) (Jussila 1984:88). 
Phyzelus nivosus Hellén

Phyzelus nivosus Hellén, 1967: 96 - Holotypus (o): "Malla",

"Reg. alp.", "Fennia", "Hellén", "965".

Gültiger Name: Arotrephes nivosus (Hellén, 1967).

\section{Phyzelus glabriculus Hellén}

Phyzelus glabriculus Hellén, 1967:96. Holotypus (o): "Parikkala Hellén", "424".

Gültiger Name: Arotrephes glabriculus (Hellén, 1967).

\section{Charitopes breviceps Hellén}

Charitopes breviceps Hellén, 1967:97f. Holotypus (૧): "Ik Ollila, 16/6 1932, K. Lahtivirta, Fennia” (Horstmann 1986:405).

Gültiger Name: Gelis breviceps (Hellén, 1967) (Horstmann 1986:405).

\section{Charitopes brevistylus Hellén}

Charitopes brevistylus Hellén, 1967:98. Holotypus (о): "Rantasalmi", "Hellén", "620" (Horstmann 1986:405).

Gültiger Name: Gelis balteata (Thomson, 1885) (Horstmann 1979b:298).

\section{Charitopes macrocerus Hellén}

Charitopes macrocerus Hellén, 1967:99. Holotypus (૧): "Kuvernööri”, "Hellén", "294".

Gültiger Name: Orthizema macrocerum (Hellén, 1967).

\section{Charitopes curticauda Hellén}

Charitopes curticauda Hellén, 1967:99. Holotypus (o): "Utsjoki Outakoski, 10.6.1947, leg. A. Saarinen, Suomi Inl."

Gültiger Name: “Ethelurgus” curticauda (Hellén, 1967).

Die Untersuchungen über die Gattungszugehörigkeit der Art sind noch nicht abgeschlossen. Die Beurteilung wird dadurch erschwert, daß beide Fühler des Holotypus schon im Jahr 1967 gefehlt haben.

\section{Isadelphus pusillus Hellén}

Isadelphus pusillus Hellén, 1967:104. Holotypus (O): "Grankulla", "Hellén", "414".

Gültiger Name: Isadelphus pusillus Hellén, 1967.

In einer früheren Publikation (Horstmann 1978:67) hatte ich diese Art zu Charitopes Förster gestellt, aber sie gehört nach Townes (in litt.) doch zu Isadelphus Förster. Die Deutung wird dadurch erschwert, daß es sich bei dem Holotypus um ein ausgesprochen kleines Exemplar handelt, wobei unbekannt ist, ob andere Individuen der Art ebenso klein sind.

\section{Isadelphus parviceps Hellén}

Isadelphus parviceps Hellén, 1967:104f. Holotypus (Q): "Fennia Le, Kilpisjärvi, V. Löfgren".

Gültiger Name: Mastrus parviceps (Hellén, 1967) (Horstmann 1978:70).

\section{Alegina thunebergi Hellén}

Alegina thunebergi Hellén, 1967:107f. Holotypus (○): "Fennia, Joutseno Sa., E. Thuneberg”, “3.5.48”.

Gültiger Name: Sulcarius thunebergi (Hellén, 1967) (Townes 1983:210).

\section{Uchidella pumila Hellén}

Uchidella pumila Hellén, 1967:108f. Holotypus (Q) "Lemland", "Hellén”, "471”.

Gültiger Name: Pleurogyrus pumilus (Hellén, 1967) (Townes 1970:45).

\section{Uchidella longicauda Hellén}

Uchidella longicauda Hellén, 1967:109. Holotypus (o): "Ik. Ollila, 26/6 1932, K. Lahtivirta, Fennia" (Horstmann 1974b:344).

Gültiger Name: Xiphulcus floricolator (Gravenhorst, 1807) (Horstmann 1974b:344).

\section{Aclastus caudator Hellén}

Aclastus caudator (Aubert als "f. aut sp.") Hellén, 1967:112. Lectotypus (○) von Horstmann (1980:141) festgelegt: "J. F. Aubert, 16.8.1961, Var, Croix Valmer" (Coll. Aubert).

Gültiger Name: Aclastus micator (Gravenhorst, 1807) (Horstmann 1979a:158f.).

Der Name "Hemiteles gracilis Ths. caudator f. aut sp. n." (Aubert 1963:868) ist eine bedingte Neubeschreibung und als solche nach den Nomenklaturregeln nicht verfügbar (Horstmann 1980:141f.). Aubert (1981:18f.) hat diese Auffassung angegriffen, ohne aber eine nach den Nomenklaturregeln zulässige andere Lösung vorzuschlagen.

\section{Aclastus setosus Hellén}

Aclastus setosus Hellén, 1967:112f. Holotypus (o): "Lemmenjoki Hellén”, "7953” (Horstmann 1980:155). 
Gültiger Name: Hedylus crassicornis Ashmead, 1899 (syn. nov.).

Bereits Townes (1983:185) hat diese Synonymisierung für möglich gehalten. Sie wurde zwischenzeitlich durch einen Vergleich des Holotypus von A. setosus mit Material von $H$. crassicornis aus der Sammlung Townes bestätigt.

\section{Aclastus opacinotum Hellén}

Aclastus opacinotum Hellén, 1967:113. Holotypus (o): "Rantasalmi", "Hellén”, "420"(Horstmann 1980:155). Gültiger Name: Orthizema opacinotum (Hellén, 1967).

Ursprünglich hatte ich diese Art mit Bedenken zu Theroscopus Förster gestellt. Sie ist mit Orthizema pullator (Gravenhorst) nah verwandt und bildet zusammen mit dieser Art innerhalb der Gattung Orthizema Förster eine eigene Artengruppe (vgl. Horstmann 1988:59f.).

\section{Aclastus planicollis Hellén}

Aclastus planicollis Hellén, 1967:113. Holotypus (Q): "Parikkala Hellén”, “4809” (Horstmann 1980:155).

Gültiger Name: Phygadeuon planicollis (Hellén, 1967) (Horstmann 1980:155).

\section{Aclastus furcifer Hellén}

Aclastus furcifer Hellén, 1967:113f. Holotypus (o): "Ivalo”, "Hellén”, "572” (Horstmann 1980:151).

Gültiger Name: Aclastus gracilis (Thomson, 1884) (Horstmann 1980:151).

\section{Catalytus fennicus Hellén}

Catalytus fennicus Hellén, 1967:114f. Holotypus (o): "Carelia or., Kuujärvi, 15.7.1943, Hellén".

Gültiger Name: Clypeoteles distans (Thomson, 1884) (Horstmann 1974a:54).

\section{Gelis cephalotes Hellén}

Gelis cephalotes Hellén, 1970:87. Holotypus (Q): "Viborg", "Hellén", "215".

Gültiger Name: Gelis cephalotes Hellén, 1970.

\section{Gelis inflatipes Hellén}

Gelis inflatipes Hellén, 1970:89. Holotypus (૧): “Terijoki. Hellén.", "179".

Gültiger Name: Gelis inflatipes Hellén, 1970.

\section{Gelis lapponicus Hellén}

Gelis inflatipes Hellén, 1970:93f. Holotypus (O): "Ivalo", "Fennia", "Hellén", "792".

Gültiger Name: Gelis lapponica Hellén, 1970.

\section{Hidryta atlantica sp. $\mathrm{n}$.}

Holotypus ( $\left.\sigma^{7}\right)$ : “Azores: Flores, Ribeira Fazenda VI. Storå", "4937”, “ơ”, "Mus. Zool. H:fors, Spec. typ. No 5922, Idiolispa morio Hellén" (Zool. Mus. Helsinki). Der Holotypus ist gleichzeitig Paralectotypus von Idiolispa morio Hellén (vgl. oben).

Beschreibungen der neuen Art finden sich unter dem Namen Idiolispa morio ( $\sigma^{7}$ nec o) bei Hellén (1967: 6) und Ortega \& Baez (1980:84f.), eine Abbildung bei Ortega \& Baez. Sie ist Hidryta pardosae Nishida ähnlich (vgl. Horstmann 1984:114). Diese weicht ab durch: Stirn und Scheitel dicht runzlig punktiert; Schläfen mäßig dicht bis dicht punktiert; Areola schmäler als lang; Nervellus etwas incliv; zweites Gastertergit beim Männchen glänzend, stellenweise mit glattem Grund.

Männchen: Schläfen hinter den Augen rundlich verengt; Gesicht fein und dicht runzlig punktiert; Stirn fein gekörnelt und fein zerstreut punktiert, stellenweise wenig gerunzelt; Fühlergruben stellenweise längsgestreift; Scheitel und Schläfen sehr fein gekörnelt, glänzend, fein und sehr zerstreut punktiert; drittes bis fünftes Fühlerglied zusammen 9.1 mal so lang wie breit, Rest der Fühler fehlend (nach Ortega \& Baez 1980:84f., Fühlergeißel 23-gliedrig, mit Tyloiden an den Gliedern 12-15); Pronotum lateral fein runzlig punktiert und längsgerunzelt; Epomia deutlich; Mesoscutum fein und mäßig dicht bis zerstreut punktiert auf glattem Grund; Notauli bis zur Mitte; Mesopleuren deutlich und sehr dicht gerunzelt; Speculum dicht punktiert, zwischen den Punkten mit schmalen glatten Zwischenräumen; Präpectalleiste dorsal bis kurz unter die Tegulae reichend; Areola so lang wie breit, nach vorn konvergierend; Ramellus deutlich; Nervellus bei 0.6 gebrochen, etwas recliv; Hinterfemora 5.5 mal so lang wie hoch; Mittelsegment kurz, abfallend, matt gerunzelt, nur im Bereich der Area superomedia weniger gerunzelt und stellenweise glänzend; vordere Querleiste kräftig; laterale und 
caudale Begrenzungen der Area superomedia undeutlich, diese etwa zweimal so breit wie lang; Stigmen 1.5 mal so lang wie breit; erstes Gastersegment dorsal basal fast glatt, median und apical gekörnelt und fein zerstreut punktiert, mit deutlich vorstehenden Stigmen; zweites Tergit fein gekörnelt und mit sehr feinen zerstreuten Haarpunkten, Stigmen um 0.5 Durchmesser von der Seitenkante entfernt.

Körper fast ganz schwarz, nur die Tibien der Vorder- und Mittelbeine basal und apical etwas aufgehellt; Pterostigma mittelbraun, Flügelfläche wenig getrübt.

Kopf $1.11 \mathrm{~mm}$ breit; Thorax $1.95 \mathrm{~mm}$ lang, $0.91 \mathrm{~mm}$ breit (Mesoscutum); Vorderflügel 3.9 $\mathrm{mm}$ lang; erstes Gastersegment $1.18 \mathrm{~mm}$ lang; Postpetiolus $0.42 \mathrm{~mm}$ lang, $0.36 \mathrm{~mm}$ breit; zweites Segment $0.89 \mathrm{~mm}$ lang, $0.88 \mathrm{~mm}$ breit; Körper etwa $5.7 \mathrm{~mm}$ lang.

Weibchen unbekannt.

Danksagungen: In erster Linie habe ich dem verstorbenen Dr. W. Hellén für seine Bereitwilligkeit zu danken, die Typen der von ihm beschriebenen Arten auch einem jungen Kollegen zur Verfügung zu stellen. Für die Zusendung weiterer Typen und anderen Vergleichsmaterials und für Auskünfte über Arten Helléns danke ich den Herren Dr. A. Albrecht und Dr. O. Biström (Zoologisches Museum der Universität, Helsinki), Dr. J.-F. Aubert (Laboratoire d'Évolution des Êtres Organisés, Paris), E. Nishida (Department of Horticulture, Ureshino, Mie, Japan), Dr. H. Townes (American Entomological Institute, Gainesville) und Drs. K. W. R. Zwart (Laboratorium voor Entomologie, Wageningen).

\section{Literatur}

Aubert, J.-F. 1963: Les Ichneumonides du rivage méditerranéen français $\left(5^{\mathrm{e}}\right.$ serie, Département du Var). - Vie et Milieu 14:847-878.

- 1969: Deuxieme travail sur les Ichneumonides de Corse (Hymenoptera). - Veröff.Zool. Staatssamml.München 13: 27-70.

- 1981: Syllogismes, illogismes et innovations chez les Ichneumonides. - Bull. Soc. Entomol. Mulhouse 1981:17-22.

Habermehl, H. 1918: Beiträge zur Kenntnis der palaearktischen Ichneumonidenfauna. - Zeitschr. Wiss. Insektenbiol. 14:145-152.

Hellén, W. 1915: Beiträge zur Kenntnis der Ichneumoniden Finlands. I. Subfamilie Pimplinae. - Acta Soc. Fauna Flora Fennica 40(6):1-89.
- 1944: Die Asyncrita-Arten Finnlands (Hym. Ichn.). Notulae Entomol. 24:11-13.

- 1949: Zur Kenntnis der Ichneumonidenfauna der Atlantischen Inseln. - Comment. Biol. 8(17):1-23.

- 1957: Zur Ichneumonidenfauna Finnlands IX (Hym.). - Notulae Entomol. 36:125-141.

- 1967: Die Ostfennoskandischen Arten der Kollektivgattungen Phygadeuon Gravenhorst und Hemiteles Gravenhorst (Hymenoptera, Ichneumonidae). - Notulae Entomol. 47:81-116.

- 1970: Die Gelis-Arten Ostfennoskandiens (Hymenoptera, Ichneumonidae). — Notulae Entomol. 50:81-94.

Horstmann, K. 1974a: Typenrevision der von Strobl in der Gattung Hemiteles Gravenhorst s. l. beschriebenen Arten und Formen (Hymenoptera, Ichneumonidae). Zeitschr. Arbeitsgem. Österr. Entomol. 25(1973):52-56.

- 1974b: Typenrevision der von E. Zilahi-Kiss beschriebenen Hemitelinen mit Bemerkungen zu den Gattungen Hemiteles Grav. (s. str.), Gnotus Foerst. und Xiphulcus Townes (Hymenoptera, Ichneumonidae). Ann. Hist.-Nat. Mus. Nat. Hung. 66:339-346.

- 1975: Zur Systematik einiger Arten der Gattung Phygadeuon Gravenhorst (Hymenoptera, Ichneumonidae). - Zeitschr. Arbeitsgem. Österr. Entomol. 26(1974):103-112.

- 1978: Revision der Gattungen der Mastrina Townes (Hymenoptera, Ichneumonidae, Hemitelinae). Zeitschr. Arbeitsgem. Österr. Entomol. 30:65-70.

- 1979a: Typenrevision der von Gravenhorst beschriebenen oder gedeuteten Hemiteles-Arten (Hymenoptera, Ichneumonidae). - Polskie Pismo Entomol. 49:151-166.

- 1979b: A revision of the types of the Hemiteles spp. described by Thomson (Hymenoptera: Ichneumonidae). — Entomol. Scand. 10: 297-302.

- 1980: Revision der europäischen Arten der Gattung Aclastus Förster (Hymenoptera, Ichneumonidae). Polskie Pismo Entomol. 50:133-158.

- 1984: Revision der paläarktischen Arten der Gattung Hidryta Förster (Hymenoptera, Ichneumonidae). Zeitschr. Arbeitsgem. Österr. Entomol. 35(1983): 113-117.

- 1986: Die westpaläarktischen Arten der Gattung Gelis Thunberg, 1827, mit macropteren oder brachypteren Weibchen (Hymenoptera, Ichneumonidae). - Entomofauna 7:389-424.

- 1988: Revision einiger westpaläarktischer Phygadeuontini (Hymenoptera, Ichneumonidae). - Nachrichtenbl. Bayer. Entomol. 37:59-64.

Jussila, R. 1979: A revision of the genus Atractodes (Hymenoptera, Ichneumonidae) in the western Palaearctic Region. - Acta Entomol. Fennica 34:1-44.

- 1984: Ichneumonidae (Hymenoptera) of Inari Lapland. - Kevo Notes 7:83-99.

Ortega, G. \& Baez, M. 1980: Contribucion al conocimiento de los Ichneumonidos de las Islas Canarias. I: Subfamilia Gelinae (Hymenoptera: Ichneumonidae). Anuar. Estud. Atlanticos 26:15-107. 
Sawoniewicz, J. 1978: Zur Systematik und Faunistik der Ichneumonidae (Hymenoptera). - Ann. Zool. 34:121-137.

- 1980: Revision of European species of the genus Bathythrix Foerster (Hymenoptera, Ichneumonidae). - Ann. Zool. 35:319-365.

Schwarz, M. 1988: Die europäischen Arten der Gattung Idiolispa Foerster (Ichneumonidae, Hymenoptera). Linzer Biol. Beitr. 20:37-66.

Seyrig, W. 1935: Faune entomologique des Iles Canaries.
Séjour de M. P. Lesne dans la Grande Canarie (1902-1903). VI. Hyménoptères Ichneumonides. Bull. Soc. Entomol. France 40:178-183.

Townes, H. 1970: The genera of Ichneumonidae. Part 2. Mem. Amer. Entomol. Inst. 12. iv + 537 pp.

- 1983: Revisions of twenty genera of Gelini (Hymenoptera). - Mem. Amer. Entomol. Inst. 35. 281 pp.

Received 8.XI.1989 